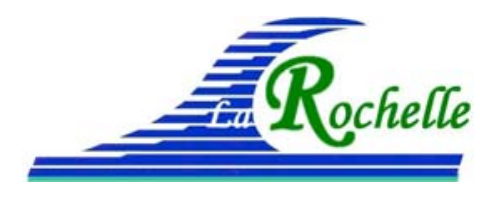

XVèmes Journées Nationales Génie Côtier - Génie Civil

La Rochelle, 29 au 31 mai 2018

DOI:10.5150/jngcgc.2018.080 (C) Editions Paralia CFL

disponible en ligne - http://www.paralia.fr - available online

\title{
Dynamiques et évolution du littoral. Synthèse des connaissances de la frontière belge à la pointe du Hourdel
}

\section{Amélie ROCHE ${ }^{1}$, Anaïs DABURON ${ }^{2}$, Alain TRENTESAUX ${ }^{3}$, Caroline MAURIN ${ }^{4}$, Yann DENIAUD ${ }^{1}$}

1. Cerema Eau, mer et fleuves, Technopôle Brest Iroise, 155 rue Pierre Bouguer, BP 5, 29280 Plouzané Cedex, France.

Amelie.Roche@cerema.fr ; Yann.Deniaud@cerema.fr

2. Cerema Nord-Picardie, 151, rue de Paris, 02100 Saint-Quentin, France. Anais.Daburon@cerema.fr

3. Université de Lille - Laboratoire d'océanologie et de géosciences, UMR 8187 LOG, Université de Lille/CNRS/ULCO, 59655 Villeneuve d'Ascq, France.

Alain.Trentesaux@univ-lille1.fr

4. DREAL Centre, 5, avenue Buffon, BP 6407, 45064 Orléans Cedex 2, France.

Caroline.Maurin@developpement-durable.gouv.fr

\section{Résumé :}

Cet article présente les travaux et principaux résultats obtenus dans le cadre de l'actualisation du "Catalogue sédimentologique des côtes françaises" sur la province sédimentaire s'étendant de la frontière belge à la pointe du Hourdel (LNH, 1986). Cette province présente trois unités morphosédimentaires : les deux plaines maritimes picarde et flamande et une côte rocheuse à falaises, le Boulonnais. Sur les dernières décennies, $40 \%$ des côtes mobiles sont en accrétion. Cependant, ce chiffre masque des secteurs où les taux de recul peuvent dépasser $3 \mathrm{~m} / \mathrm{an}$. Par ailleurs, environ $60 \%$ de ce littoral est impacté par près de $130 \mathrm{~km}$ d'ouvrages et aménagements côtiers, dont certains, bloquant les sédiments transportés par la dérive littorale vers le nord-est, définissent les limites des huit cellules hydrosédimentaires. Ces transports sédimentaires correspondent essentiellement à la remobilisation d'un matériel hérité, non renouvelé, car les faibles apports actuels sont liés au recul des côtes à falaises et aux sédiments transportés depuis la province méridionale adjacente. L'élévation actuelle du niveau de la mer laisse craindre une fragilisation d'espaces littoraux déjà contraints et de fortes tensions sur les capacités d'adaptation des habitats naturels côtiers. Ces impacts sont toutefois soumis à de nombreuses incertitudes et les efforts de mesure et de capitalisation des connaissances doivent être poursuivis pour adopter des stratégies de gestion durables.

\section{Mots-clés :}

Dynamique sédimentaire, Érosion, Évolution du trait de côte, Gestion durable du littoral, Sédiments, Ouvrages côtiers, Aménagement du littoral, Changement climatique. 


\section{Thème 6 - Gestion durable des zones littorales et estuariennes}

\section{Introduction}

La collection "Dynamiques et évolution du littoral" rassemble et synthétise les connaissances disponibles pour identifier et mettre en évidence les points essentiels explicatifs de l'évolution du littoral français. Elle correspond à l'actualisation du "Catalogue sédimentologique des côtes françaises" sur la métropole, dont le premier fascicule concerne la partie septentrionale des côtes françaises (LNH, 1986), et intègre les territoires ultramarins. Ces documents traduisent l'état des connaissances actuelles et évaluent la sensibilité du littoral aux dérèglements climatiques en cours; ils pointent également les secteurs et thématiques où les connaissances sont faibles ou en voie d'approfondissement. Avec près de 500 pages illustrées de 75 cartes inédites, le premier fascicule de cette collection (CEREMA, 2018) traite de la province sédimentaire s'étendant de la frontière belge à la pointe du Hourdel (baie de Somme) sur environ $240 \mathrm{~km}$. Également appelée côte d'Opale, cette province se caractérise par la diversité de ses paysages (grandes plages sableuses ourlées de massifs dunaires, côtes rocheuses escarpées, estuaires), ses stations balnéaires (Le Touquet-Paris-Plage, Berck-sur-Mer, Wimereux...) et ses infrastructures portuaires (Boulogne-sur-Mer, Calais et Dunkerque pour les plus importantes). Cet article revient sur les travaux réalisés entre 2013 et 2017 et présente les principaux résultats obtenus, notamment sur les aspects de connaissance des mouvements sédimentaires et de l'évolution du trait de côte, des impacts anthropiques et des perspectives d'évolution dans un contexte de changement climatique. Des voies de prolongement des travaux de connaissances sont identifiées en fin d'article. Le lecteur est invité à consulter le document et son atlas cartographique en ligne sur les sites Internet du Cerema (http://www.cerema.fr) et du Géolittoral (http://www.geolittoral.developpement-durable.gouv.fr/) pour compléter les informations de ce résumé et obtenir toutes les références nécessaires.

\section{Méthode d'élaboration}

\section{1 Étude de faisabilité et organisation globale du projet}

L'historique et les objectifs du projet d'actualisation du "Catalogue sédimentologique" figurent dans ROCHE et DENIAUD (2014). L'opportunité du projet a été confirmée par une enquête entre avril et juin 2011, accessible sur Internet à partir des sites Géolittoral et du Cetmef. Ses résultats ont été synthétisés dans ROCHE et al. (2011) et traduits dans une étude de faisabilité (2011-2012), précisant la forme et le fond du nouveau document, ainsi que ses modalités d'organisation et de production. Le projet, inscrit dans le programme d'actions 2012-2015 de la Stratégie nationale de gestion intégrée du trait de côte (SNGITC), a été validé par le Ministère de l'écologie, du développement durable et de l'énergie fin 2012. Le Cerema a été chargé de sa mise en œuvre et des référents locaux, coordonnateurs de comités de rédaction locaux, ont été nommés (voir ROCHE \& DENIAUD, 2014). 


\section{XVèmes Journées Nationales Génie Côtier - Génie Civil \\ La Rochelle, 29 au 31 mai 2018}

Un comité de coordination associant l'ensemble des référents locaux a été réuni chaque année afin de garantir un traitement harmonisé des informations techniques, tout en respectant les spécificités territoriales. L'avancement du projet a été par ailleurs régulièrement présenté aux instances de pilotage de la SNGITC et validé sur un plan scientifique et technique par le comité scientifique de l'axe A de la stratégie nationale, présidé par M. Stéphane COSTA, professeur de géographie à l'université de Caen.

\subsection{Contributeurs locaux}

Une réunion de lancement du projet sur la province s'est tenue le 22 octobre 2013 à la station marine de Wimereux et a réuni 21 personnes issues de 17 organismes locaux. Consolidé au fur et à mesure du projet, le comité de rédaction a rassemblé au final plus de 60 personnes issues de 34 organismes ; elles ont été sollicitées pour l'écriture et la relecture du document entre 2013 et 2017. La rédaction du document a principalement été assurée en interne par le Cerema, avec un rédacteur principal désigné par chapitre en fonction des compétences techniques requises. Cette méthodologie a permis d'aboutir rapidement à une première version de chaque chapitre, soumise à la relecture au comité de rédaction dès 2014. Afin d'harmoniser les contributions reçues des relecteurs, des réunions de travail focalisées sur des chapitres ont été organisées dans les locaux de l'université de Lille à Villeneuve d'Ascq en novembre 2015 et octobre 2016. En amont de ces réunions, le comité de rédaction avait été sollicité pour la relecture des documents avec un délai de retour d'au moins 5 semaines. Bien que la plupart des compléments rédactionnels et ajouts de données bibliographiques complémentaires soient parvenus par mail, chacune de ces réunions a été bénéfique au projet car elle relançait les discussions avec les contributeurs locaux. Malgré les prises de contact avec les organismes locaux et les nombreux échanges avec eux, l'exhaustivité des données bibliographiques et des contributeurs impliqués reste difficile à évaluer complètement.

\subsection{Exploitation des données et études disponibles localement}

Les recherches bibliographiques menées par le Cerema dès 2013 ont été complétées par les partenaires locaux au fur et à mesure de leurs relectures jusqu'en 2016. Cette bibliographie initiale a été enrichie chaque année par des études scientifiques et techniques menées pour des questions de gestion locale (études ponctuelles liées à des projets d'aménagement, travaux de rechargement des plages, etc.) ou en fonction des évènements naturels (tempêtes, érosions ponctuelles, etc.). Au final, 874 références ont été recensées et exploitées pour l'établissement du document (voir figure 1).

Aux références bibliographiques s'ajoutent les données géomatiques: 137 couches d'informations géographiques ont été créées spécifiquement pour le projet par le Cerema et sont disponibles dans un visualiseur cartographique dynamique sur le site Internet Géolittoral. Elles ont permis l'établissement de 75 cartes thématiques citées dans le texte et regroupées dans un atlas cartographique indépendant. 


\section{Thème 6 - Gestion durable des zones littorales et estuariennes}

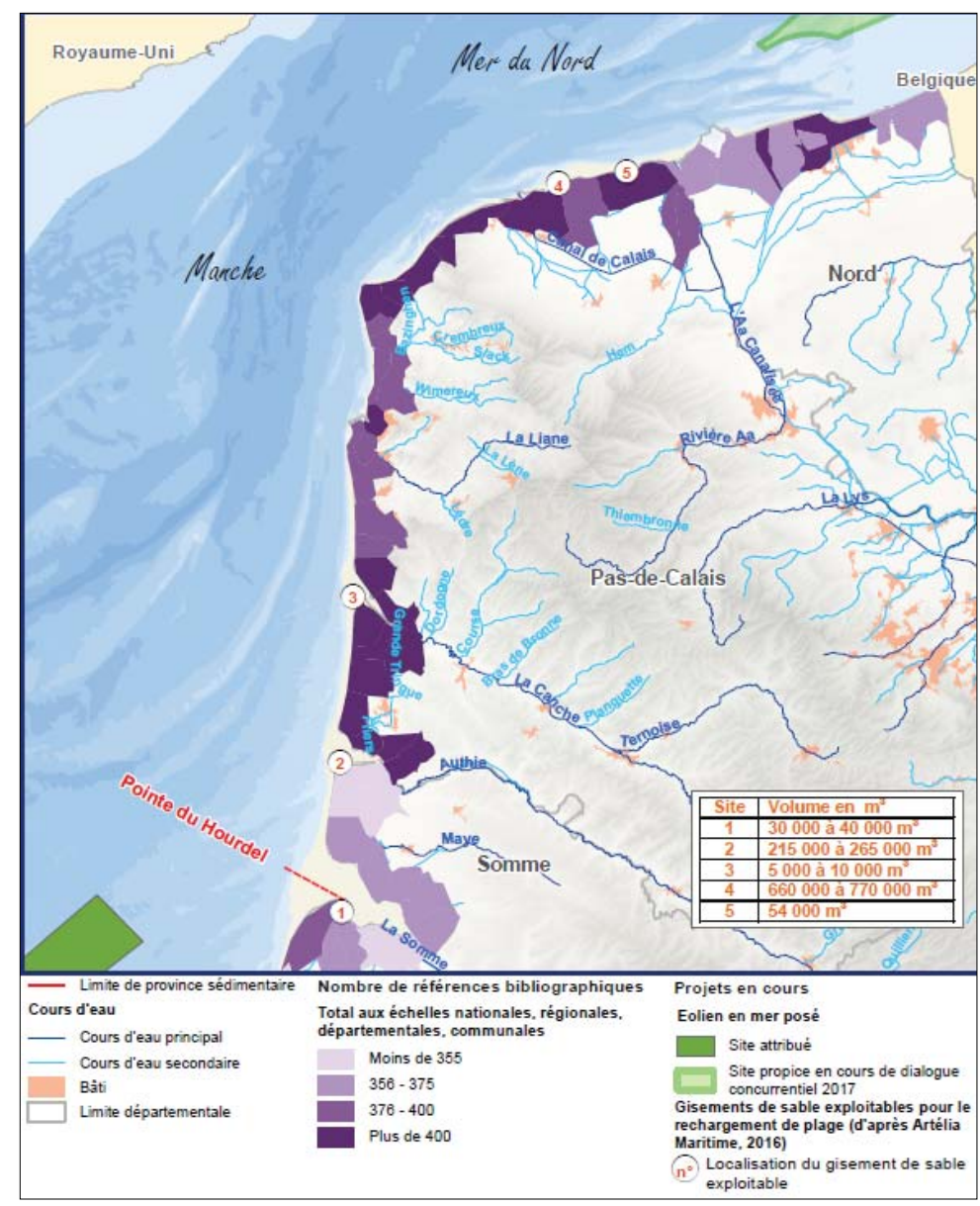

Figure 1. Références bibliographiques disponibles et projets en cours sur la province sédimentaire de la frontière belge à la pointe du Hourdel (CEREMA, 2018).

\section{Principaux résultats}

Les études menées sur la province sont très nombreuses, mais d'échelles temporelles et spatiales très diverses : certains travaux d'envergure nationale (Shom, Ifremer...) sont ponctuellement complétés par des études locales. Les zones considérées "à risques" sont ainsi souvent mieux renseignées (travaux nombreux et régulièrement mis à jour). Mais la proximité géographique d'un centre de recherche (études nombreuses et/ou menées à plus long terme) ou la survenue d'un sinistre peuvent également expliquer l'abondance d'informations sur certains territoires. La disponibilité des données bibliographiques reste contrainte par la volonté de diffusion des producteurs et par une accessibilité restreinte, parfois liée à la simple méconnaissance de leur existence. Depuis le catalogue (LNH, 1986), la province a été étudiée par AUGRIS et al. (1990), inspirant la réalisation du Plan Littoral d'Actions pour la Gestion de l'Érosion sur le littoral de la Côte d'Opale -PLAGE- (SMCO, 2003). Les travaux menés plus récemment au niveau national dans le cadre de la SNGITC ont également permis d'accroître la connaissance 


\section{XVèmes Journées Nationales Génie Côtier - Génie Civil \\ La Rochelle, 29 au 31 mai 2018}

de l'évolution du trait de côte et des ouvrages et aménagements côtiers (CEREMA, 2015).

\subsection{Connaissance de l'évolution du trait de côte}

Les tendances d'évolution du trait de côte à moyen et long terme sont bien appréhendées grâce aux levés du trait de côte réalisés à partir d'orthophotographies anciennes et complétés sur la période récente par des levés de terrain. L'indicateur national de l'érosion côtière (CEREMA, 2015) permet notamment d'évaluer les vitesses moyennes d'évolution du littoral de la province sur plus de 70 ans mais aussi de les comparer aux autres provinces sédimentaires françaises. Le trait de côte de la province évolue peu à long terme, avec des périodes d'érosion pouvant être suivies de périodes d'engraissement. Sur les dernières décennies, $40 \%$ des côtes mobiles de la province sont en accrétion, un des taux les plus élevés de métropole, notamment au niveau de Oye-Plage (120 mètres gagnés sur 1934-2009) ou à l'est de Dunkerque (plus de 13 mètres gagnés sur 1963-2000). Cependant, ce chiffre masque des différences très marquées, avec des taux de recul du trait de côte pouvant dépasser localement $3 \mathrm{~m} / \mathrm{an}$ en moyenne (notamment en baie d'Authie ou de Wissant). En effet, les zones les plus évolutives correspondent aux estuaires dont la migration des ensembles sédimentaires suit le sens de la dérive littorale : une accrétion généralisée des rives gauches se traduit par un développement important de flèches sableuses alors que des phénomènes érosifs en rive droite ont souvent donné lieu à des travaux de confortement.

L'évolution des littoraux est également marquée par des événements météorologiques tempétueux qui peuvent provoquer des désordres ou des reculs significatifs ponctuels du trait de côte. Récemment, les événements de l'hiver 2013-2014 ont ainsi provoqué des mouvements de terrain, en particulier à Equihen-Plage avec un recul en crête de falaise mesuré de 6 mètres en 2013. La variabilité des taux d'évolution du trait de côte souligne ainsi la présence d'une dynamique beaucoup plus locale et la nécessité d'étudier la côte d'Opale à plusieurs échelles spatiales.

\subsection{Connaissance des mouvements sédimentaires}

Les mouvements de sédiments sur la province, déjà bien documentés dans le précédent document (LNH, 1986), ont été précisés voire quantifiés. Ils correspondent essentiellement à la redistribution d'un matériel hérité, non renouvelé, mis en place lors de la transgression holocène et remanié. En effet, les apports sédimentaires actuels sont faibles et principalement liés au recul des côtes à falaises et aux sédiments transportés depuis la province méridionale adjacente. Les sédiments sont transportés longitudinalement par la dérive littorale orientée vers le nord pour la côte de la Manche et vers l'est pour la côte de la mer du Nord. Ce transport sédimentaire résultant vers le nord-est est en accord avec l'asymétrie des courants tidaux orientés vers le nord, associée à des houles dominantes de provenance ouest à sud-ouest. L'estran de la baie 


\section{Thème 6 - Gestion durable des zones littorales et estuariennes}

de Wissant constitue un cas particulier avec une inversion locale de la dérive littorale résiduelle orientée ouest-sud-ouest, observée d'Escalles au cap Gris-Nez.

Les estrans de la province présentent un système typique de barres-bâches. Les larges estrans découverts à marée basse par un important marnage sont essentiels pour alimenter les cordons dunaires, bien que ces échanges perpendiculaires au rivage ne soient pas aisément quantifiables et restent relativement méconnus, notamment au nord de la province (voir figure 2). Ils sont cependant capitaux, en particulier sur le littoral de la mer du Nord, en raison de l'important stock sédimentaire représenté par les grands bancs de sable. Des accolements à la côte de ces structures kilométriques sont documentés par des cartes historiques. Ils contribuent ainsi à la progradation du littoral.

Les massifs dunaires, baies et estuaires constituent des puits de sédiments temporaires, lieux de dépôt et de stockage provisoires pouvant réalimenter le système naturellement lors de modifications significatives des facteurs de forçage, en lien par exemple avec l'impact du changement climatique. Les baies et les estuaires de la province captent une grande partie des apports fluviatiles et présentent une concentration élevée de particules fines. Par ailleurs, la quantité considérable de sable stockée dans les petits fonds, en particulier au nord de la province, pourrait servir de source sédimentaire importante pour le maintien et l'entretien de la position du trait de côte.

Sur la province, les limites des 8 cellules hydrosédimentaires sont bien établies dans la littérature existante. Ces limites sont liées aux "épis hydrauliques" constitués par les estuaires de la côte d'Opale, au cap Gris-Nez et aux jetées portuaires des 3 principaux ports qui bloquent la majeure partie du transit sédimentaire. Seule la frontière belge est une limite fictive : les sédiments transportés par la dérive littorale sortent du système français et continuent vers le nord-est de la mer du Nord (voir figure 2).

Les travaux menés par le Cerema dans le prolongement de l'indicateur national de l'érosion côtière (CEREMA, 2015) ont permis de recenser les ouvrages et aménagements côtiers susceptibles d'influencer le trait de côte. La province cumule ainsi près de $130 \mathrm{~km}$ linéaires d'ouvrages et aménagements côtiers, impactant près de $60 \%$ de son littoral. Les premiers aménagements connus, et parfois encore visibles aujourd'hui sur le littoral ou l'espace rétro-littoral de la province, datent du Moyen Âge. Leurs principaux objectifs étaient alors d'augmenter les surfaces agricoles et d'assécher les zones humides pour limiter les problèmes sanitaires (phase de poldérisation). Le développement du tourisme et l'urbanisation littorale ont débuté à la fin du XIX ${ }^{\mathrm{e}}$ siècle et se sont poursuivis au $\mathrm{XX}^{\mathrm{e}}$ siècle avec l'apparition de stations balnéaires comme Le Crotoy, Berck-sur-Mer, Le Touquet-Paris-Plage, Wimereux ou Malo-les-Bains. Parallèlement, l'essor de la vie maritime au $\mathrm{XIX}^{\mathrm{e}}$ a permis le développement économique des ports de Calais, Boulogne-sur-Mer et Dunkerque, et l'augmentation de travaux d'entretien pour les chenaux d'accès par la réalisation de dragages. 


\section{XVèmes Journées Nationales Génie Côtier - Génie Civil \\ La Rochelle, 29 au 31 mai 2018}

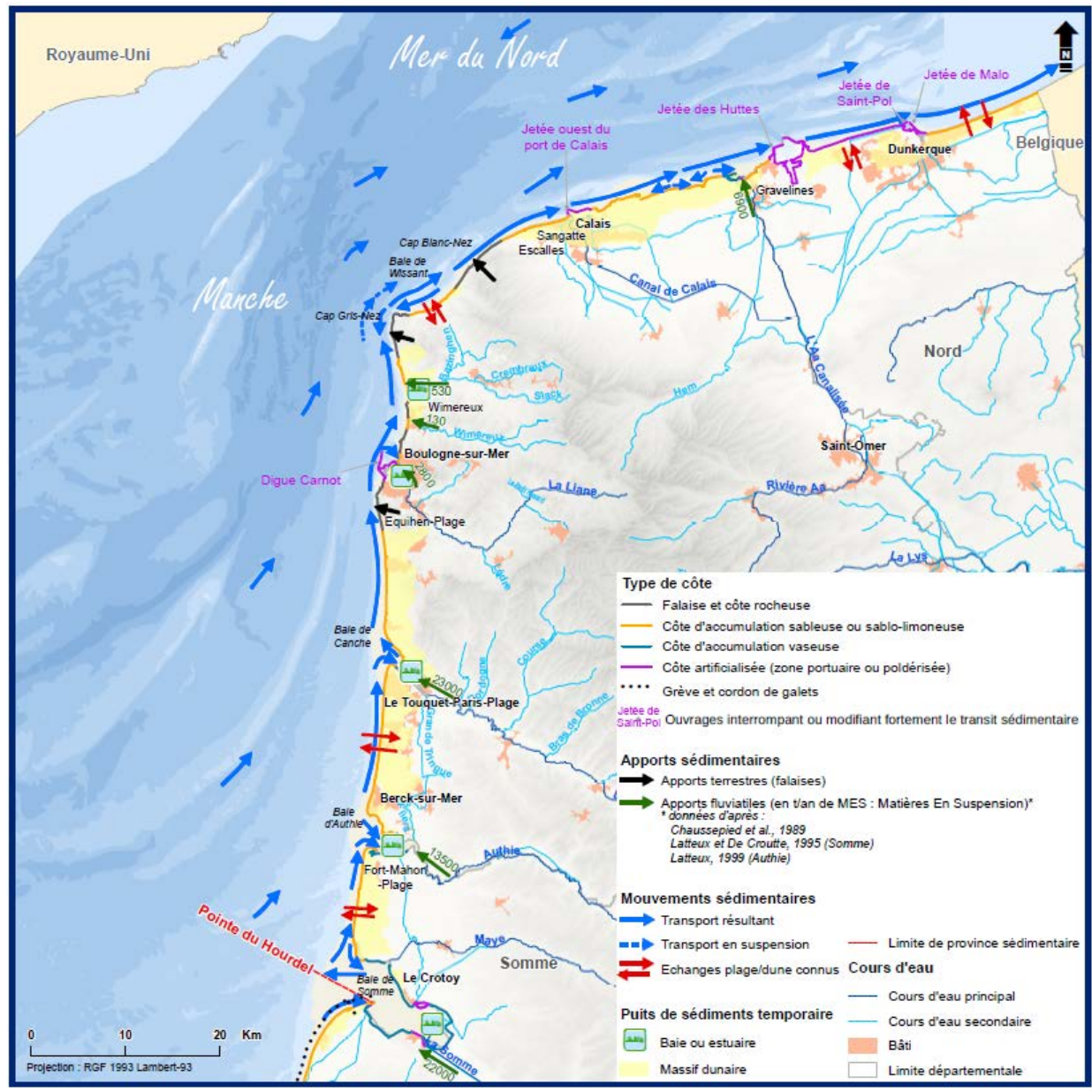

Figure 2. Bilan sédimentaire actuel sur la province sédimentaire de la frontière belge à la pointe du Hourdel (CEREMA, 2018).

Ces développements économique et touristique augmentent de fait les enjeux présents sur le territoire, le rendant plus vulnérable aux aléas littoraux comme le recul des falaises rocheuses dans le Boulonnais ou les submersions marines et inondations dans les zones basses des plaines maritimes picarde et flamande. L'augmentation de ces enjeux s'est longtemps accompagnée du renforcement et de la construction d'ouvrages de défense contre la mer : contre les submersions marines (digues), pour le maintien du trait de côte (perrés et murs) et contre l'érosion des côtes meubles (épis, brise-lames). Des portes à la mer ont également été réalisées, principalement dans les polders, pour contrôler les entrées d'eau dans les terres. Ces constructions ont ainsi bloqué une part des sédiments d'origine terrestre. Les estuaires, qui font partie des zones les plus évolutives de la province, voient l'évolution contrastée de leurs deux rives contrariée depuis un siècle par les aménagements portuaires ou touristiques. Les efforts de recherche doivent être poursuivis sur ces zones afin d'accompagner les modifications naturelles et notamment celles liées aux évolutions climatiques attendues. 


\section{Thème 6 - Gestion durable des zones littorales et estuariennes}

\section{Perspectives et conclusion}

Le travail réalisé a permis de dresser un état des connaissances actualisé sur la province s'étendant de la frontière belge à la pointe du Hourdel, avec des références plus nombreuses et plus précises en lien avec l'évolution des techniques d'observation et de modélisation. L'évolution du littoral et des fonds y est relativement bien documentée dans la littérature scientifique et technique: les cellules hydrosédimentaires sont délimitées et les mouvements sédimentaires globaux bien appréhendés. La connaissance de l'évolution du trait de côte et des ouvrages et aménagements côtiers, déjà bien établie localement, a également bénéficié des travaux menés au niveau national dans le cadre de la SNGITC. Cependant, un approfondissement de la connaissance est encore possible sur certaines parties de ce territoire moins étudiées ou sur des thématiques émergentes, notamment les transports sédimentaires dans le profil de plage (échanges plage/dune), la variabilité interannuelle de la dérive littorale ou encore les relations entre les bancs de sable au large et l'évolution du trait de côte. Autre exemple, l'impact du changement climatique global présente encore de fortes incertitudes aux échelles locales. La part des effets des activités anthropiques reste délicate à évaluer par rapport à celle des forçages globaux et la capacité d'adaptation des systèmes naturels à ces modifications extérieures demeure incertaine et reste également à évaluer.

L'évolution des techniques d'acquisition telles que les levés aéroportés par LiDAR ou drone, terrestres voire bathymétriques, et le développement des applications satellitaires laissent entrevoir des potentialités plus fortes à l'avenir pour mieux suivre l'évolution du littoral, avec de meilleures résolution et fréquence d'acquisition. Cette multiplication des données pose cependant les questions de leur capitalisation et de leur mise à disposition, voire de leur qualification et de leur pérennité sur le long terme. Le réseau national des observatoires du trait de côte, également inscrit dans le programme d'actions de la SNGITC, vise notamment à mieux faire connaître, partager et diffuser la connaissance sur les territoires ; en filigrane, il permettrait également à terme de faciliter le partage de documents ou de données de référence actualisées sur l'évolution du trait de côte. Par ailleurs, le transfert de cette connaissance technique vers les acteurs locaux, avec un accompagnement approprié, est nécessaire pour l'élaboration de stratégies locales durables sur le littoral.

\section{Références bibliographiques}

AUGRIS C., CLABAUT P., VICAIRE O. (1990). Le domaine marin du Nord-Pas de Calais - Nature, morphologie et mobilité des fonds. Ifremer/Conseil régional du Nord Pas de Calais. 96 p. + cartes.

CEREMA (2015). Indicateur national de l'érosion côtière. 


\section{XVèmes Journées Nationales Génie Côtier - Génie Civil \\ La Rochelle, 29 au 31 mai 2018}

CEREMA (2018). Dynamiques et évolution du littoral. Synthèse des connaissances des côtes de la frontière belge à la pointe du Hourdel. 498 p. + atlas cartographique 84 p.

LNH -LABORATOIRE NATIONAL D'HYDRAULIQUE- (1986). Catalogue sédimentologique des côtes françaises - Côtes de la Mer du Nord et de la Manche. Partie A: de la frontière belge à la baie de Somme. Secrétariat d'État auprès du Ministre de l'urbanisme, du logement et des transports, chargé de la mer. Direction des ports et de la navigation maritimes. Service technique central. Ed. Eyrolles. Direction des études et recherches d'Électricité de France, pp 11-133

ROCHE A., DENIAUD Y. (2014). Actualisation du "Catalogue sédimentologique des côtes françaises". Journées Nationales Génie Côtier - Génie Civil, Dunkerque, pp 205214. http://dx.doi.org/10.5150/jngcgc. 2014.023

ROCHE A., TRMAL C., LEBAILLEUX A. (2011). Analyse des résultats de l'enquête sur l'actualisation des catalogues sédimentologiques des côtes françaises. CETMEF, $54 \mathrm{p}$.

SMCO -SYNDICAT MIXTE DE LA CÔTE D'OPALE- (2003). Plan Littoral d'Actions pour la Gestion de l'Érosion sur le littoral de la Côte d'Opale. 186 p. 
Thème 6 - Gestion durable des zones littorales et estuariennes 